\title{
Darius Reinhardt
}

\section{Vattenfall vs. Deutschland (II) und das Internationale Investitionsschutzregime in der Kritik}

Im Mai 2012 hat das schwedische Unternehmen Vattenfall Europe AG bekanntgegeben, gegen den am 6. Juni 2011 beschlossenen stufenweisen Atomausstieg Deutschlands zu klagen. Im Gegensatz zu den zwei weiteren AKW-Betreibern E.ON und RWE geht Vattenfall dabei nicht den Weg über das Bundesverfassungsgericht, sondern klagt, wie schon im Streit um die Umweltbestimmungen des Kohlekraftwerkes Hamburg Moorburg, vor dem International Center for the Settlement of Investment Disputes (ICSID) in Washington.

Im Folgenden möchte ich einen Überblick über die Klage Vattenfalls geben, bevor ich einen kritischen Blick auf die Rolle der Menschenrechte werfe.

Dabei stellt sich die Frage, was das ICSID ist und wieso Vattenfall im Gegensatz zu E.ON und RWE die Möglichkeit hat, gegen ein deutsches Gesetz in Washington zu klagen. Daraufhin werden die Grundlagen der Klage vor dem ICSID bestimmt, auf denen die Argumentation Vattenfalls basieren könnte. Abschließend werde ich darauf eingehen, wie Menschenrechte in Zukunft stärker berücksichtigt werden könnten.

\section{Investitionsschutzverträge und Schiedsgerichte}

„Bilateral Investment Treaties“ (BIT's) bilden den Rahmen für Verfahren vor dem ICSID. Bilaterale Investitionsverträge, so die Annahme, dienen dazu, die jeweilige wirtschaftliche Entwicklung der Vertragsparteien zu stärken, indem sie ausländische Direktinvestitionen fördern ${ }^{1}$ und indem die Verträge „[...] getätigte Investitionen vor gewissen politischen Risiken schützen [...], um so die Bereitschaft zu Auslandsinvestitionen zu erhalten“.2

Die Geschichte der BIT's reicht bis in das Jahr 1959 zurück, als Deutschland den ersten BIT mit Pakistan unterzeichnete. Heute ist Deutschaland mit über 150 in Kraft getretenen Verträgen weltweit führend. ${ }^{3}$

Seit den 1980ern haben BIT's einen regelrechten Boom erlebt. Die sogenannten Schiedsklauseln erlauben es den Investoren, wie im Falle Vattenfall, vor einem Schiedsgericht gegen Staaten zu klagen, Happ und Bischoff bezeichnen dies als ein „denationalisiertes“ Verfahren. ${ }^{4}$ Investoren nutzen diese Möglichkeit zunehmend um gegen staatliches Handeln vorzugehen. Die überwiegende Zahl der Verfahren führt zu einer Entscheidung zulasten der Staaten (im Jahr 2013 wurden bisher $70 \%$ der veröffentlichten Schiedssprüche zugunsten der Investoren ent-

1 UNCTAD, 2000, S. iii, abrufbar: http://unctad.org/en/Docs/poiteiiad2.en.pdf.

2 Bischoff/Happ, "Hilfe, ich werde enteignet! - Abkommen schützen Auslandsinvestitionen", 2011, S. 2, abrufbar unter: http://www.gtai.de/GTAI/Content/DE/Trade/Fachdaten/PUB/2011/08/pub2011 08268001_16060.pdf.

3 Bruno Simma, Foreign Investment Arbitration: A Place for Human Rights?, International \& Comparative Law Quarterly : ICLQ Vol. 60, No. 3, 8, 11, 2011, S. 574.

4 Bischoff/Happ (Fn. 2), S. 14, 
schieden)..$^{5}$ Es ist davon auszugehen, dass ein weit größerer Teil der Konflikte

zwischen Investoren und Staaten unter dem Druck eines drohenden Schiedsverfahrens bereits vor der Anrufung eines Schiedsgerichtes zu einer Einigung kommen und dass der drohende Schatten des „Investor State Dispute“ (ISD) zu einem „Regulatory Chill“ führt. ${ }^{6}$ Welche Konsequenzen dies für Sozial- und Umweltstandards hat, werde ich abschließend erläutern.

Das ICSID stellt heute den meistgenutzten Rahmen für die ISD's dar. ${ }^{7}$ Es handelt sich bei dem ICSID jedoch weder um ein Gericht noch um ein Schiedsgericht. Vielmehr bietet der ICSID den Streitparteien einen organisatorisch-institutionellen und verfahrensrechtlichen Rahmen. Kommt es zu einem Schiedsverfahren, werden ad-hoc Tribunale berufen. Dabei wird von den Konfliktparteien je ein Vertreter ausgewählt, bevor diese beiden sodann gemeinsam einen Schiedsrichter wählen (Art. 37 ICSID-Konvention).

Die Klage Vattenfalls basiert auf dem Energy Charter Vertrag (EnCV). Der EnCV ist seit April 1989 in Kraft und ist im Gegensatz zu den üblichen BIT's multilateral, sektorspezifisch und regional. ${ }^{8}$ Der EnCV ist heute wohl die meistgenutzte Grundlage für Investitionsschutzstreitigkeiten. ${ }^{9}$ Vattenfall erhofft sich mit seiner Klage im Rahmen des ICSID wesentliche Vorteile gegenüber einer Beschreitung des nationalen Rechtsweges.

Zunächst dürfen die Streitparteien nach Artikel 12-16 ICSID-Konvention die Schiedsrichter selber aus dem in Washington geführten Schiedsrichterverzeichnis wählen, was für den Ausgang des Verfahrens von erheblicher Bedeutung sein kann.

Ferner besteht für Vattenfall der große Vorteil darin, dass das ICSID zu Entschädigungszahlungen verpflichten kann und dass eine solche Entscheidung jeglicher staatlicher Revision entzogen wäre. Das Bundesverfassungsgericht hingegen könnte in dem vorliegenden Fall nur feststellen, dass der Atomausstieg gegen die Verfassung verstößt. Entschädigungen kämen schon deshalb nicht in Frage, da das deutsche Recht sogenannte „de-facto Enteignungen“ nicht kennt. Als defacto oder regulative Enteignung werden Maßnahmen bezeichnet, die einer Enteignung des Investors gleichkommen. ${ }^{10}$ Genau darum könnte es sich im Falle der Entscheidung zur Verkürzung der AKW-Laufzeiten handeln. Eine „de-facto Enteignung“" müsste nach Art. 13 ECT „angemessen“ und „unverzüglich“ entschädigt werden. Vattenfall beziffert seinen Verlust durch den Atomausstieg auf rund 3,7 Milliarden Euro.

\section{Vattenfall und die Klage vor dem ICSID}

Im Hinblick auf den Inhalt der Klage bzw. die Argumentation lassen sich nur Vermutungen anstellen. Regelmäßig werden weder Schiedsspruch, Verfahrensverlauf noch Klageschrift („,Request for Arbitration“) der ICSID-Verfahren veröffentlicht. So führte auch eine Kleine Anfrage der Opposition im Bundestag

IIA-Issues Note, UNCTAD, Recent Developments in Investor - State Dispute Settlement (ISDS), 2013, abrufbar unter: http://unctad.org/en/PublicationsLibrary/webdiaepcb2013d3_en.pdf.

Simma (Fn. 3), S. 578 f.

Bischhoff/Happ (Fn.2), S. 13 f. sowie IIA-Issues Note (Fn. 5), S. 1.

Krajewski, Vattenfall, der deutsche Atomausstieg und das internationale Investitionsrecht, Juridikum 2013, S. 354.

9 Krajewski(Fn. 8), S. 3.

10 Krajewski (Fn. 8), S. 4 f. 
nicht dazu, dass Inhalte der Klageschrift bekannt gemacht wurden. ${ }^{11}$ Vielmehr wurde auf das laufende Verfahren und die den Regeln des ICSID entspringende Verpflichtung zur Vertraulichkeit verwiesen. Dieser Ansicht widerspricht ein Offener Brief von Peter Fuchs im Namen des PowerShift e.V. und wirft der Bundesregierung die bewusste Entscheidung vor, der Öffentlichkeit Informationen über das Verfahren vorzuenthalten. ${ }^{12}$ Der Antwort auf die Kleine Anfrage ist zu entnehmen, dass die Bundesregierung dem Parlament in der Geheimschutzstelle des Deutschen Bundestages einen Bericht zur Einsichtnahme ausgelegt hat.

Im Folgenden soll dennoch versucht werden, die mögliche Argumentation nachzuzeichnen. Dies geschieht vor allem unter Rückgriff auf Erfahrungen aus vergangenen Prozessen. Als Grundlage für diesen Abschnitt wird vor allem der im Juni 2012 erschienene Bericht „Der deutsche Atomausstieg auf dem Prüfstand eines internationalen Investitionsschiedsgerichts?" von Nathalie BernasconiOsterwalder und Rhea Tamara Hoffmann (2012) dienen. ${ }^{13}$

Das Verfahren scheint bereits ordnungsgemäß eingeleitet, da die Bundesregierung bereits am 21. Dezember 2011 von Vattenfall gemäß Art. 26 (2) EnCV „eine Aufforderung zur gütlichen Beilegung" erhalten hat. Nach der vorgeschriebenen dreimonatigen Verhandlungsfrist zur gütlichen Beilegung eines Streits kann der Investor gemäß Art. 26 (2) a) bis c) EnCV nicht nur eine Beilegung des Streits vor einem Zivil- oder Verwaltungsgericht anstreben, sondern hat auch die Möglichkeit, sich an das ICSID oder das Institut für Schiedsverfahren der Stockholmer Handelskammer zu wenden. ${ }^{14}$ Am 31. Mai 2012 reichte Vattenfall den ISD-Antrag vor dem ICSID ein.

Voraussichtlich wird sich die Vattenfall Europe AG in ihrer Klage maßgeblich auf drei Regelungen des ECT beziehen:

a) auf den Schutz vor Enteignung,

b) auf die Pflicht zur fairen und gerechten Behandlung und

c) auf die sogenannte Schirmklausel, die Pflicht, allen Verpflichtungen gegenüber Investoren nachzukommen.

\section{a) Enteignungsschutz}

Gemäß Art.13 ECT dürfen Investitionen von ausländischen Investoren nicht verstaatlicht werden, sofern diese nicht durch eine angemessene Entschädigungsleistung kompensiert werden. Wichtig ist hierbei vor allem der Wortlaut des Artikels. Demnach dürfen Investitionen nicht „Maßnahmen gleicher Wirkung wie Verstaatlichung oder Enteignung unterzogen werden“. Derartige Maßnahmen gleicher Wirkung werden als „De-Facto Enteignungen“ oder „Indirekte Enteignungen“ bezeichnet. Läge ein solcher Tatbestand vor, müsste Deutschland Vattenfall „angemessen“ und „unverzüglich“ entschädigen. ${ }^{15}$

11 Deutscher Bundestag, Antwort der Bundesregierung auf die Kleine Anfrage der Abgeordneten Sylvia Kotting-Uhl, Harald Ebner, Hans-Josef Fell, weiterer Abgeordneter und der Fraktion BÜNDNIS 90/ DIE GRÜNEN, BT-Drs. 17/10584, 28.8.2012.

12 Fuchs, Offener Brief an die Mitglieder des Ausschusses für Umwelt, Naturschutz und Reaktorsicherheit im Deutschen Bundestag, 22.6.2012, abrufbar unter: http://power-shift.de/wordpress/wp-content/ uploads/2012/06/Powershift-Brief_Umweltausschuss-Vattenfall-ICSID-22-6-12.pdf.

13 Bernasconi-Osterwalder/Hoffmann, Der deutsche Atomausstieg auf dem Prüfstand eines internationalen Investitionsschiedsgerichts?", 2012, abrufbar unter: http://power-shift.de/wordpress/wp-content/uploads/2012/06/Bernasconi-Hoffmann-Vattenfall-ICSID-Briefing-PowerShift-Sept2012.pdf.

14 Fogt Bergby, Grundlagen und aktuelle Entwicklungen im Streitbeilegungsrecht nach dem Energiechartavertrag aus norwegischer Perspektive, Halle (Saale) 2003, abrufbar unter: http://telc.jura.uni-halle.de/sites/default/files/altbestand/Heft23.pdf, S. $11 \mathrm{ff}$.

15 Für eine kritische Diskussion der „De-Facto Enteignung “: Mair/Mildner, Im Schulterschluss für offene Märkte und Investorenschutz, in: SWP-Aktuell 2013/A 15, Februar 2013, S. 7 f. 
Vattenfall wird argumentieren, dass die Aufhebung der in der 11. Atomgesetznovelle vorgesehene Verlängerung der Laufzeiten einer Enteignung gleich kommt. Anders gesagt: dass die verlängerte Betriebsgenehmigung, welche hier als Eigentum gewertet werden könnte, mit dem Atomausstieg wertlos geworden sei. Fraglich ist jedoch, ob das Schiedsgericht den Tatbestand der indirekten Enteignung als erfüllt ansieht. Regelmäßig lassen sich drei unterschiedliche Herangehensweisen der Investitionsschiedsgerichte beobachten:

- Sollte sich das Gericht vorrangig auf die Folgen staatlicher Maßnahmen konzentrieren, wäre die Absicht, die zu der Maßnahme führte, irrelevant. Es würde demnach keinen Unterschied machen, ob ein Staat die Maßnahme ergriff, um beispielsweise die nationale Wirtschaft zu bevorzugen, oder zum Schutz von Menschenrechten agierte.

- Einen Mittelweg würde eine Abwägung zwischen dem Nutzen einer Maßnahme für das Gemeinwohl und dem Schaden für den Investor darstellen. Eine solche Abwägung kann mithin sehr komplex sein, da auch Urteile über die Angemessenheit und Effektivität der zur Disposition stehenden Maßnahme mit einbezogen werden müssten.

- Als drittes könnte das Gericht die staatlichen Maßnahmen nur im Hinblick auf das Gemeinwohl bewerten und daher ungeachtet ihrer Wirkung den Vorgang nicht als Enteignung werten. Die Praxis zeigt jedoch, dass diese Frage selten so regulierungsfreundlich interpretiert wird.

Es ist nicht möglich vorherzusehen, welchem Ansatz sich das Schiedsgericht anschließen wird. Dies hängt maßgeblich von zwei Faktoren ab: a) der Zusammensetzung des Schiedsgerichts und b) dem speziellen Sachverhalt.

Diese Frage zielt auf den Kern der Kritik an den Investitionsschutzabkommen. Ein transparentes System würde Klarheit sowohl für Investoren als auch für Staaten schaffen. Ein solches System müsste die internationalen Verpflichtungen von Staaten mit einbeziehen, da es ansonsten keinen realistischen Investitionsschutz bieten kann und Staaten in einem Spannungsfeld zurücklässt, in dem sie sich die Einhaltung ihrer Menschenrechtsverpflichtungen durch „angemessene Entschädigungen“ erkaufen müssen. ${ }^{16}$

\section{b) Faire und gerechte Behandlung}

Ein weiterer Grundsatz der meisten Investitionsschutzverträge ist die gerechte und billige Behandlung („fair and equitable treatment“, FET). So verpflichtet auch Art. 10 (1) EnCV jede Vertragspartei dazu, „stabile, gerechte, günstige und transparente Bedingungen“ für Investoren anderer Vertragsparteien zu fördern und zu schaffen. Auch dieser Grundsatz wurde von den Schiedsgerichten sehr unterschiedlich interpretiert. Zumeist folgten die Interpretationen jedoch dem Sinne des Zieles der Abkommen: dem Schutz von Investitionen.

In diesem Falle bleibt zu beachten, dass der EnCV in seiner Präambel Bestimmungen und Zielformulierungen zum Umweltschutz einbezieht. Vattenfall wird sich darauf berufen, dass es infolge der 11. Atomgesetznovelle vor dem Hintergrund „legitimer Erwartungen“ 700 Millionen Euro in die beiden Kernkraftwerke Krümmel und Brunsbüttel investierte. ${ }^{17}$ Genau diese „legitimen Erwartungen“ könnten durch den deutschen Atomausstieg verletzt worden sein. Gleichzeitig leitete die Opposition kurz nach der 11. Atomgesetznovelle ein

17 Balser, Vattenfall verklagt Regierung auf Entschädigung Atomausstieg ja, aber bitte mit Kompensation, Die Zeit v. 5.6.2012, abrufbar unter: http://www.sueddeutsche.de/wirtschaft/vattenfall-verklagt-regierung-auf-entschaedigung-atomausstieg-ja-aber-bitte-mit-kompensation-1.1374584. 
Normenkontrollverfahren vor dem Bundesverfassungsgericht ein. Das Schiedsgericht könnte dies dahingehend auslegen, dass nie ein Vertrauensschutz entstanden sei. ${ }^{18}$

Ferner könnte sich Vattenfall im Sinne des Art. 10 Abs. 1 ECT auf das Verbot ungleicher und diskriminierender Behandlung berufen, da nicht alle Unternehmen von der Wirkung der 13. Atomgesetznovelle in gleicher Weise betroffen sind. ${ }^{19}$ Dass bereits die Möglichkeit der Klage vor dem ICSID E.ON und RWE als inländische Investoren diskriminiert, spielt hier keine Rolle.

\section{c) Die Schirmklausel}

Der letzte Satz des Art. 10 (1) EnCV eröffnet Vattenfall eine weitere Möglichkeit, die Verletzung seiner Rechte geltend zu machen. Dieser verpflichtet den Gaststaat dazu, „alle Verpflichtungen, die ein Gaststaat gegenüber einem Investor oder einer Investition eines Investors einer anderen Vertragspartei eingegangen ist, zu erfüllen“. Auf Basis dieses weit auslegbaren Artikels könnte Vattenfall geltend machen, dass die im September 2010 vereinbarten Laufzeitverlängerungen eine Verpflichtung für Deutschland darstelle, die durch den Atomausstieg verletzt wurde. Interessanterweise haben einige Staaten bei den Verhandlungen zu dem ECT den letzten Satz des Art. 10 Abs. 1 explizit ausgeschlossen. Bernasconi-Osterwalder und Hoffmann nehmen an, dass diesen Staaten die „Internationalisierung sämtlicher Verpflichtungen“ zu weit ging; die Bundesrepublik Deutschland gehört nicht zu diesen Staaten.

\section{Internationale Schiedsgerichtsbarkeit und Menschenrechte}

Die Diskussion über das Verfahren Vattenfall vs. Deutschland (II) ist erst am Anfang. Dies liegt vor allem daran, dass Informationen über das Verfahren kaum öffentlich zugänglich sind. Es ist dringend nötig, dass in Zukunft eine Praxis der Transparenz Einzug in die Verfahren der ICSID speziell und internationaler Schiedsgerichte im Allgemeinen findet. Das die Bundesregierung jegliche Stellungnahme zu einem Verfahren verweigert, das die Bundesrepublik 3,7 Milliarden Euro kosten könnte und für dessen Verhandlung die Bundesregierung im kommenden Jahr 2,2 Millionen Euro bereitstellt, ${ }^{20}$ folgt der Intransparenz des Verfahrens an sich. Abgesehen davon, dass dies an sich verfassungsrechtlich bedenklich ist, könnte man die Frage stellen, inwieweit diese Geheimhaltungspolitik den bereits besprochenen „Chilling Effect“ verstärkt. Wenn Parlamentarier bei ihren Entscheidungen die Informationen über die völkerrechtlichen Implikationen ihrer Entscheidungen vorenthalten bleiben, könnte dies aus Angst vor einem drohendem Schiedsverfahren zu einem Trend wesentlich restriktiveren Regierungshandelns gegenüber Investoren führen. Dieser Trend würde zum Nachteil von Menschenrechts- und Umweltstandards verlaufen.

Verstößt der Atomausstieg gegen internationale Investitionsschutzabkommen?, 21.3.2012, abrufbar unter: http://www.derenergieblog.de/alle-themen/energie/verstost-der-atomausstieg-gegen-internationale-investitionsschutzabkommen/.

19 Wie Deutschland aus der Atomkraft aussteigt, Stern.de vom 5. Juni 2011, Abrufbar unter: http:// www.stern.de/politik/deutschland/fahrplan-zur-energiewende-wie-deutschland-aus-der-atomkraftaussteigt-1692466.html.

20 Werdermann, Der verklagte Atomausstieg, Der Freitag, 2013, abrufbar unter: http://www.freitag.de/ autoren/felix-werdermann/der-verklagte-atomausstieg 834-2014-1-86 
Transparenz kann nur der erste Schritt zu einer öffentlichen Diskussion über den

Zweck und die Gefahren von Investitionsschutzabkommen sein. Die Anzahl derartiger Abkommen ist in den letzten Jahren stetig gestiegen, und die Möglichkeit zum ISD ist immer häufiger genutzt worden. Anders gesagt, die Rechte von Investoren wurden umfangreich geschützt, während es zu negativen Auswirkungen auf Umwelt oder Menschenrechte gekommen ist. Wo aber liegen die Ursachen dafür, dass Umwelt und Menschenrechtsstandards nur selten Eingang in die Schiedssprüche des internationalen Investitionsschutzes finden? Die asymmetrische Interessenvertretung von Investor-Interessen ist kein Zufall. Die Grundlagen des heutigen Investitionsschutzregimes können bis in das 17. Jahrhundert zurückgeführt werden. Während die Anfänge des Systems zwischen europäischen Nationen auf Augenhöhe stattfanden, wandelte sich der Charakter des Investitionsschutzes im Zuge des Kolonialismus schnell zu einem Machtinstrument. ${ }^{21}$ Die Verträge ermöglichten es den europäischen Kolonialmächten, ihre territorialen und wirtschaftlichen Bestrebungen in ein scheinbar neutrales Gewand zu kleiden.22 Die zugrunde liegenden Ziele sind bis heute dieselben geblieben. Weiterhin bilden Ressourcen- und Machtasymmetrien die Grundlage für Investitionsschutzverträge. ${ }^{23}$ Auch im 21. Jahrhundert folgt die Systematik der Investitionsschutzverträge einseitig einer ökonomistischen Logik und stellt den Schutz der ausländischen Investoren über den Schutz von Menschenrechten, der Umwelt und des Gemeinwohls.

Ein möglicher Weg zur Stärkung der Responsivitätspflichten der Schiedsgerichte könnte in einer engeren Rückbindung an die staatlichen Rechtsordnungen und ihre Legitimationsmuster liegen. Doch in welchem Verhältnis steht nationales Recht und die internationale Schiedsgerichtsbarkeit? Hinsichtlich dieser Frage besteht Uneinigkeit in der Rechtslehre; jedoch lassen sich zwei konkurrierende Theorien ausmachen. ${ }^{24}$ Die "jurisdiktionell-prozessrechtliche Theorie" 25 geht davon aus, dass sich die Autorität der internationalen Schiedsgerichtsbarkeit aus der nationalen Rechtsprechung ableite. Die „autonome Theorie“ hingegen betont die Loslösung der internationalen Schiedsgerichtsbarkeit von nationalen Rechtsordnungen. ${ }^{26}$ Folgt man diesem Ansatz, so entspringt die Autorität der internationalen Schiedsgerichtsbarkeit der Autonomie der Vertragsparteien. Moshe Hirsch verweist darauf, dass der Investitionsschutz in zwei Schritten erfolgt. ${ }^{27}$ Zum einen wird die Beziehung zwischen Staat und Investor durch den Abschluss von Investitionsschutzverträgen auf die privatrechtliche Dimension herabgesenkt, während gleichzeitig der Schutz derselben auf die international Ebene angehoben wird, um die Einhaltung der Verträge durch das Völkerrecht abzusichern. In der Praxis führt das dazu, dass allein über die Rückbindung der Schiedsgerichtsbarkeit an die Nationalstaaten kaum gerechte Ergebnisse erzielt werden können. Denn die Klagen von Investoren zielen primär auf die privatrechtlichen beziehungsweise investitionsrechtlichen Gesichtspunkte $a b$, während der Schiedsspruch weitgehende Auswirkungen auf Dritte und deren Men-

24 Renner, Zwingendes transnationales Recht: zur Struktur der Wirtschaftsverfassung jenseits des Staates, 2001, S. 76.

25 Schlosser, Das Recht der internationalen privaten Schiedsgerichtsbarkeit, 1989, Rn. $40 \mathrm{ff}$.

26 Renner (Fn. 24), S. 76.

27 Hirsch, Investment Tribunals and Human Rights: Divergent Paths, in: Dupuy/Francioni/Petersmann, Human Rights in International Investment Law and Arbitration, 2009, S. 114. 
schenrechte hat. ${ }^{28}$ Dies hat zur Folge, dass die internationale Schiedsgerichtsbarkeit einseitig die Interessen der Investoren vertritt und damit die Souveränität der Staaten im Hinblick auf gemeinwohlorientierte Regulierungen untergräbt. So ist es in den vergangenen Jahren immer häufiger zu Jurisdiktionskonflikten gekommen, die durch internationale Schiedsgerichte regelmäßig zu Lasten des Gemeinwohls entschieden wurden. Dies liegt vor allem daran, dass die Schiedssprüche völkerrechtlich bindend sind und dass Staaten, abgesehen von den streng limitierten Revisionsverfahren, innerhalb der internationalen Schiedsgerichtsbarkeit keine Möglichkeit haben, die Entscheidungen anzufechten.

Viele Autoren haben in der Vergangenheit darauf hingewiesen, dass Menschenrechtsstandards stärkere Berücksichtigung in internationalen Investitionsschutzverfahren erfahren müssen. ${ }^{29} \mathrm{~W}$ ie könnte also eine Harmonisierung von Menschenrechten und Investitionsschutz aussehen? Bruno Simma hat dazu in seinem Artikel „Foreign Investment Arbitration, A Place for Human Rights?“ zwei grundsätzliche Ansätze vorgeschlagen. Zum einen könnten Menschenrechte auf zwischenstaatlicher Ebene direkt in den Investitionsschutzverträgen verankert bzw. bei deren Interpretation als Rechtsquelle herangezogen werden. ${ }^{30}$ Der Konsens scheint hier zu sein, dass Art. 31 Abs. 3 Buchst. c WVK es ermögliche, Menschenrechten als Völkerrechtssätze bei der Auslegung von Investitionsschutzverträgen heranzuziehen. ${ }^{31}$

Eine weitere Möglichkeit bestünde darin, bei dem Abschluss konkreter Investitionsverträge die Menschenrechtsverpflichtungen des Staates zu berücksichtigen. ${ }^{32}$ Dies würde den Investoren erlauben, das Verhalten des Staates besser einschätzen zu können und so die Investitionssicherheit besser bestimmen zu können, während der Staat aus dem unbefriedigenden Spannungsverhältnis entlassen würde. Konkret schlägt Simma ein „Human Rights Audit”33 vor. So könnte bereits im Vorfeld abgewogen werden, ob die Erwartungen des Investors durch den Staat im Rahmen seiner Menschenrechtsverpflichtungen erfüllt werden können, um einem möglichen Jurisdiktionskonfliktaus dem Weg zu gehen. Ein solcher Mechanismus könnte gewissermaßen als das Äquivalent zu dem „Enviromental Impact Assessment“ (EIA) gesehen werden, der die Berücksichtigung von Umweltstandards bereits vor der Autorisierung von Investitionen evaluiert. ${ }^{34}$ Dazu fügt sich, dass selbst Autoren wie Petersmann die mangelnde Transparenz und die gravierenden Auswirkungen der internationalen Schiedsgerichtsbarkeit auf öffentliche Interessen betonen und vorschlagen, die defizitäre Berücksichtigung von Menschenrechten durch deren Aufnahme in den institutionellen Rahmen der WTO ausgleichen. ${ }^{35}$

Doch diese Ansätze greifen zu kurz. Sie führen, wie Philip Alston es formuliert hat, zu einem „Merger and Acquisition of Human Rights“, weil diese Rechte in den „WTO/ICSID and ...“-Ansätzen den ökonomistischen Logiken unterge-

Siehe: Reiner/Schreuer, Human Rights and International Investment Arbitration, in: Dupuy/Francioni/ Petersmann, Human Rights in International Investment Law and Arbitration, 2009, S. 93, sowie: Hirsch (Fn. 26), S. 114.

29 Franzki/Horst, Der Fall Chevron: Das emanzipatorische Potential des Rechts in der doppelten Fragmentierung des globalen Rechts, i.E. S. 12.

30 Simma (Fn. 3), S. 580 f.

31 Franzki/Horst (Fn. 28), S. 12.

32 Simma, Fn.3, S. 592 f.

33 Simma (Fn. 3), S. $573 \mathrm{ff}$.

34 Pavoni, Environmental Rights, Sustainable Development, and Investor-State Case Law: A Critical Appraisal, in: Hirsch, Investment Tribunals and Human Rights: Divergent Paths, in: Dupuy/Francioni/ Petersmann, Human Rights in International Investment Law and Arbitration, 2009, S. 545 f.

35 Siehe: Petersmann, International Rule of Law and Constitutional Justice in International Investment Law and Arbitration, 16 Ind. J Global Legal Stud. 2009. 
ordnet werden. Die WTO garantieren zwar ökonomische Freiheiten, allerdings seien diese nicht annähernd mit der Tradition der internationalen Menschenrechte zu vergleichen. ${ }^{36}$ Alston zufolge würde die WTO in Petermanns Ansatz den sozialen Rechten effektiv keinen Raum einräumen, ${ }^{37}$ vielmehr würden die Menschenrechte im Sinne der Wirtschaft instrumentalisiert:

"Their purpose is fundamentally different. Human rights are recognized for all on the basis of the inherent human dignity of all persons. Trade-related rights are granted to individuals for instrumentalist reasons. Individuals are seen as objects rather than as holders of rights. They are empowered as economic agents for particular purposes and in order to promote a specific approach to economic policy, but not as political actors in the full sense and nor as the holders of a comprebensive and balanced set of individual rights. "38

Das Ziel des internationalen Investitionsschutzrechtes ist selbstredend der Schutz ausländischer Investitionen. Dies scheint evident zu sein, allerdings heben einige Autoren hervor, dass damit auch die Semantik sowie die Rationalität des Investitionsschutzrechts aus einer spezifisch ökonomischen Basis erwächst. ${ }^{39}$ Diese spezifische Rationalität ist kein Einzelfall, sondern Ausdruck einer Entwicklung, die zu einer fortschreitenden Fragmentierung des Völkerrechts ${ }^{40}$ und zu einer Vielzahl von Regimen, die sich jeweils nur auf ihren spezifischen Sektor beziehen, führt. Durch diese Fragmentierung ist die Kollision zwischen den Regimen vorprogrammiert. Auf dieser Grundlage ist ein „ökonomischer Bias“ der internationalen Schiedsgerichtsbarkeit zu erkennen, deren Selbstverständnis von Investmentschutz geprägt ist. Die effektive Einbeziehung oder Abwägung anderer „Bereichsrationalitäten“ ist daher regelmäßig nur innerhalb des inhärenten neoliberalen Paradigmas möglich. ${ }^{41}$ Dieser These folgt auch der Ansatz Petermanns, Menschenrechte im Rahmen der WTO zu verankern, der sich unter Alstons Analyse als Wolf im Schafspelz oder Investorenschutz im Menschenrechtskleid herausgestellt hat.

Daraus folgern Franzki und Horst, dass eine Berücksichtigungspflicht von Menschenrechten im internationalen Investitionsschutz nicht ausreichend sein kann. Eine solche würde einer Kompetenzerweiterung der internationalen Schiedsgerichtsbarkeit gleichkommen und die bestehenden Asymmetrien verfestigen. ${ }^{42}$

Vielmehr bedürfe es einer „[...] interne[n] Selbstbegrenzung der Jurisdiktion der Schiedsgerichte für Sachverhalte mit menschenrechtlichem Drittbezug [...]”. ${ }^{43}$ Gemeinsam mit dem von Simma vorgeschlagenen „Human Rights Audit“ könnte dieser Ansatz nicht nur einen adäquaten Menschenrechtsschutz für durch die Investition Betroffene bieten, sondern auch die Investitionssicherheit für Investoren stärken.

Leider hat die akademische Debatte zu dem Thema bis jetzt wenig Früchte getragen. Vattenfall vs. Deutschland II könnte zu einem entscheidenden Momentum in Deutschland werden, um die Debatte in die Öffentlichkeit zu tragen. Fokus der Kritik muss hier zu allererst das nicht zu rechtfertigende Transparenzdefizit sein. Wenn ein Schiedsgericht des ICSID darüber entscheidet, ob Deutschland 3,7 Milliarden Euro Kompensationszahlungen aus öffentlichen

36 Alston, Resisting the Merger and Acquisition of Human Rights by Trade Law: A Reply to Petersmann, in 13 European Journal of International Law, 2002, S. 826.

Alston (Fn. 36), S. 827.

Alston (Fn. 36), S. 826.

Franzki/Horst (Fn. 29), S. 14 f.

Hierzu grundlegend Andreas Fischer-Lescano/Gunther Teubner, Regime-Kollisionen, 2006.

Franzki/Horst (Fn. 29), S. 16.

Franzki/Horst (Fn. 29), S. 23.

Franzki/Horst (Fn. 29), S. 17 
Geldern zu zahlen hat, haben die Bürgerinnen und Bürger ein Recht darauf, über die Einzelheiten des Verfahrens aufgeklärt zu werden. Illegitimer Weise folgt die Praxis hier der Logik des Privatrechts, während es sich bei dem Streitpunkt um ein öffentliches Anliegen handelt, welches die Öffentlichkeit seit Jahrzehnten beschäftigt.

\section{Justiz und Komödie}
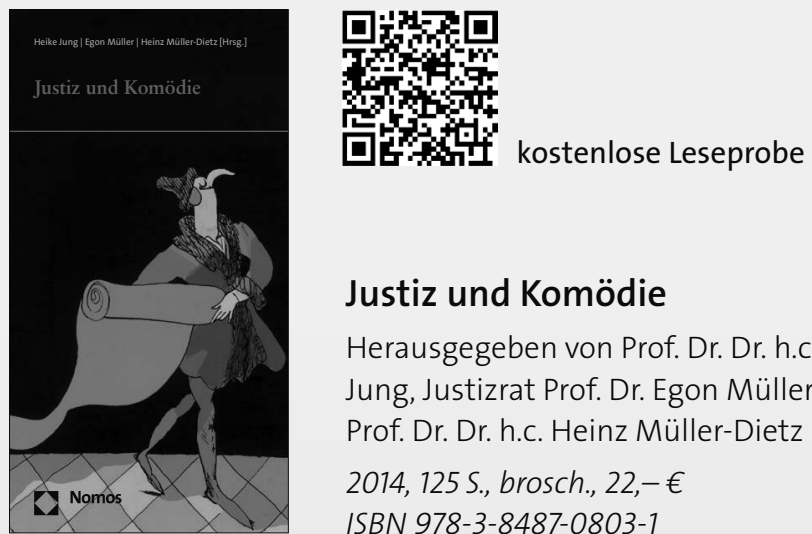

\section{Justiz und Komödie}

Herausgegeben von Prof. Dr. Dr. h.c. Heike Jung, Justizrat Prof. Dr. Egon Müller und Prof. Dr. Dr. h.c. Heinz Müller-Dietz

2014, 125 S., brosch., 22,- $€$

ISBN 978-3-8487-0803-1

Wer sich Zugang zu „Recht und Justiz“ verschaffen will, sollte nicht nur juristische Lehrbücher, Kommentare und Gerichtsentscheidungen zur Hand nehmen. Die Literatur bietet eine andere, bisweilen sogar erhellendere Sicht auf die Grundprobleme und -konflikte von Recht und Justiz.

Das Genre der Komödie eignet sich in besonderer Weise dazu, der Justiz den Spiegel vorzuhalten. Die Werkanalysen des Bandes belegen dies mit Kostproben aus der Weltliteratur der Komödie.

Der Reigen umfasst zwei französische Komödien („Maître Pathelin“ und „Les Plaideurs“), Kleists „Der zerbrochne Krug“, Nestroys Possen und türkische Kadi-Komödien nebst einer Art Prolog über Sprache und Recht. Mit seinem Schwerpunkt auf der Komödie setzt der Band in dem breiteren Spektrum von „Literatur und Recht" einen eigenen unverwechselbaren Akzent.

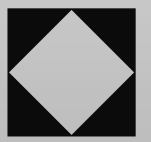

